\title{
Die Freiheit, Kippen zu verkaufen ...
}

\section{Samia Hurst}

Dr. med., Institut für Biomedizinische Ethik, Medizinische Fakultät, Genf

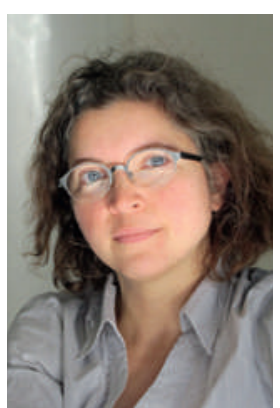

Gemäss dem Bundesamt für Gesundheit sterben in der Schweiz jedes Jahr fast 10000 Personen vorzeitig an den Folgen eines Nikotinabusus. Die Weltgesundheitsorganisation schätzt, dass auf der Basis des heutigen Tabakkonsums die Nikotinsucht bis 2030 die weltweit häufigste Todesursache sein wird. Aus Sicht der Medizin sind die Dinge schon seit langem absolut klar: Rauchen ist gesundheitsschädigend und lebensgefährlich, und zwar nicht nur für die Raucher selbst, sondern auch für ihr Umfeld. Nach fast einem Jahrhundert hartnäckigen Ignorierens der Fakten [1] musste selbst die Tabakindustrie zugeben, dass diese Zusammenhänge bestehen. In diesem Kontext erhalten die Diskussionen um das Tabakproduktegesetz im Hinblick auf die Gesundheit grosses Gewicht.

In Bezug auf die Schweizer Wirtschaft ist die Nikotinsucht offensichtlich auch von grosser Bedeutung. Sie beeinflusst Arbeitsverhältnisse, Steuern, Finanzierungen durch die Industrie. Es ist deshalb zu erwarten, dass jeglicher Versuch, den Tabakkonsum mittels Gesetz einzuschränken, diese Interessen beschneiden wird. Auf globaler Ebene kann diese Konfrontation ein eindrückliches Ausmass annehmen. Wollen Staaten ihre Bevölkerung mittels Einschränkung des Zigarettenkonsums schützen, werden sie seit einiger Zeit durch die Tabakindustrie fast gewohnheitsgemäss vor Institutionen gebracht, die ein wirtschaftliches Pendant zu internationalen Ge-

\section{Der Konflikt spielt sich hier zwischen zwei gesellschaftlichen Kräften ab.}

richtshöfen darstellen. Profitstreben ist ab jetzt, so scheint es, etwas Strafbares geworden. Der Konflikt spielt sich hier zwischen zwei gesellschaftlichen Kräften ab: der Gesundheitsförderung und der Gewinnförderung.

Es überrascht also nicht, dass der Gesetzesentwurf zum Tabakproduktegesetz in der Schweiz auf Widerstand stösst. Weil der Konflikt zwischen Gesundheit und Wirtschaftlichkeit Unbehagen auslöst, wird oft ein bequemeres Argument bemüht. Wenn Zweifel über die
Berechtigung eines Entwurfs zum Tabakproduktegesetz bestehen, so fussen sie nicht nur auf rein monetären Überlegungen. Nein, es geht hier um die Frage der Freiheit. Freiheit ist wichtig. Wer dies nicht so sieht, soll die Hand erheben. Niemand? Da sehen Sie es ... Jedoch muss etwas klargestellt sein: Es geht hier nicht um die Freiheit des Rauchers. Die meisten Raucher wurden während ihrer Adoleszenz zu Rauchern. Es gibt bessere Beispiele für die freie Wahl erwachsener Menschen. Ausserdem geht es nicht darum, die Zigarette zu verbieten. Jeder hat immer noch die freie Wahl zu rauchen. Nein, die Freiheit, die hier verteidigt wird, ist die Freiheit der Tabakindustrie. Es ist die Freiheit, ein gefährliches und suchterzeugendes Produkt zu fördern und daraus Gewinn zu ziehen. Was auch verstanden werden sollte: Es geht um den Wettstreit zwischen der Freiheit von Unternehmen und der Freiheit von

Es geht um den Wettstreit zwischen der Freiheit von Unternehmen und der Freiheit von Personen.

Personen. Bei Tabakprodukten müssen wir eine Wahl treffen: Wenn wir dem Hersteller mehr Freiheit gewähren, seine Produkte zu fördern, beschränken wir dadurch die Möglichkeiten des Bürgers, frei von der Zigarette zu sein. Diese Schlussfolgerung ist keine theoretische. Das ausdrückliche Ziel der Förderung von Tabakprodukten ist die Erhöhung des Konsums. Werbung zu verbieten, reduziert den Konsum [2] und damit die Anzahl abhängiger Personen, die mit dem Rauchen aufhören möchten [3]. Wessen Freiheit ist uns also wichtig? Dies ist die eigentliche Frage.

\section{Literatur}

1 Brandt A. The Cigarette Century: the Rise, Fall, and Deadly Per sistence of the Product that Defined America. New York: Basic Books; 2009.

2 Saffer H, Chaloupka F. The effect of tobacco advertising bans on tobacco consumption. Journal of health economics. 2000;19(6):1117-37.

3 Siahpush M, Yong HH, Borland R, et al. Smokers with financial stress are more likely to want to quit but less likely to try or succeed: findings from the International Tobacco Control (ITC) Four Country Survey. Addiction. 2009;104(8):1382-90. 